\title{
Large falcine meningioma fed by callosomarginal branch successfully removed following contralateral interhemispheric approach
}

\author{
Ramon F. Barajas Jr. • Michael E. Sughrue • \\ Michael W. McDermott
}

Received: 14 March 2009/Accepted: 10 August 2009/Published online: 29 August 2009

(C) The Author(s) 2009. This article is published with open access at Springerlink.com

\begin{abstract}
We report the case of a highly vascular facline meningioma removed following surgical ligation of a large callosomarginal feeding branch via a contralateral interhemispheric approach. Successfully addressing this vessel via a contralateral interhemispheric approach prior to any debulking allowed for en bloc Simpson Grade 1 tumor removal with minimal blood loss and short term tumor control without evidence of recurrence at 2 year follow up. A 56 year old man presented with first time generalized tonic-clonic seizure. Imaging revealed a right sided $5 \mathrm{~cm}$ falcine meningioma. The patient underwent pre-operative embolization of feeding branches, however, the most significant supply, arising from the right callosomarginal artery, could not be occluded. A bipartite frontotemporal craniotomy was performed. From a left sided interhemispheric approach the pericallosal and callosomarginal arteries were identified and the large callosomarginal tumor feeding branch were occluded using a straight Yasargil aneurysm clip. From the right the superior sagital sinus was ligated anteriorly and posteriorly. The sinus, falx, and adherent tumor were then removed en bloc. We present the case of a highly vascular falcine meningioma with a large callosomarginal feeding branch which was successfully occluded using surgical clipping of this vessel via a contralateral interhemispheric approach. This case provides an excellent example of one approach to directly dealing with
\end{abstract}

Electronic supplementary material The online version of this article (doi:10.1007/s11060-009-9994-1) contains supplementary material, which is available to authorized users.

R. F. Barajas Jr. · M. E. Sughrue · M. W. McDermott ( $\bowtie)$ Department of Neurological Surgery, University of California at San Francisco, 505 Parnassus Ave M779, Box 0112,

San Francisco, CA 94117, USA

e-mail: McDermottM@neurosurg.ucsf.edu large, deep interhemispheric feeding vessels unsuitable for embolization. A 3D animation of the surgical approach is provided for instructional purposes.

Keywords Falcine meningioma $\cdot$ Callosomarginal artery $\cdot$ Contralateral interhemispheric approach

\section{Introduction}

The goal of surgical intervention in meningiomas is complete resection and removal of dural attachments. Often attainment of this goal is complicated by factors such as tumor location, extent of dural attachments, and the complexity of tumor blood supply. Falcine meningiomas are often richly supplied by multiple terminal arterial branches of the middle meningeal artery, callosomarginal artery, pericallosal artery, and branches of the anterior falx artery which can complicate any surgical attempts at resection [3].

Feeding arteries can be located medial or inferior to the tumor making control of bleeding difficult until the tumor is removed $[1-3,8]$. When the blood supply is predominantly pial and the artery is distal within the vascular tree, embolization can be difficult or impossible without significant risk. Surgical approaches to the main blood supply of tumors vary by location and size. Accessing the feeding artery on the inferior surface of a large tumor attached to an immobile membrane such as the falx is very difficult without extreme retraction or corticectomy. One option is a contralateral approach to the blood supply via the interhemispheric fissure.

We report the case of a highly vascular meningioma with a large callosomarginal feeding artery successfully removed following surgical clipping of this vessel via a contralateral interhemispheric approach. Successfully 
addressing this vessel prior to any debulking allowed for en bloc Simpson Grade 1 tumor removal with minimal blood loss and short term tumor control without evidence of recurrence at 2 year follow up.

\section{Clinical presentation}

Presentation

The patient is an otherwise healthy 59 year old man who presented to the University of California San Francisco emergency department following a first time generalized tonic-clonic seizure. Imaging performed after arrival demonstrated a right sided $5 \mathrm{~cm}$ homogenously enhancing extra-axial falcine mass with an adjacent cystic component suggestive of meningioma (Fig. 1).

The patient underwent preoperative angiography. The external carotid artery blood supply, from the superficial temporal and middle meningeal arteries, was embolized bilaterally. The more significant supply, however, arose from a large right callosomarginal artery, which was not able to be safely embolized. The large contrast jet directly into the tumor from this artery suggested that this feeding vessel needed to be successfully addressed prior to any tumor debulking (Fig. 2).

\section{Surgical approach}

One week following embolization the patient was brought to the operating room for tumor resection. After placement of a lumbar subarachnoid drain, the patient was positioned supine and the head was pinned bitemporally with the neck slightly flexed in a Mayfield head holder (Fig. 3a). A bi-coronal scalp incision was made posterior to the tumor. Due to the vascularity and extradural supply of this tumor the cranial vault was opened via bi-partite bone flap (Fig. 3b): A right frontoparietal craniotomy with boundaries just above the superior temporal line laterally and just short of midline medially was turned first, followed by a left frontoparietal craniotomy crossing the midline and extending just medial to the superior temporal line.

The dura was then opened in a U-shaped fashion based medially on the left. Two small parasagital draining veins were cauterized and divided. Using gentle retraction on the medial left frontal lobe, the interhemispheric fissure was
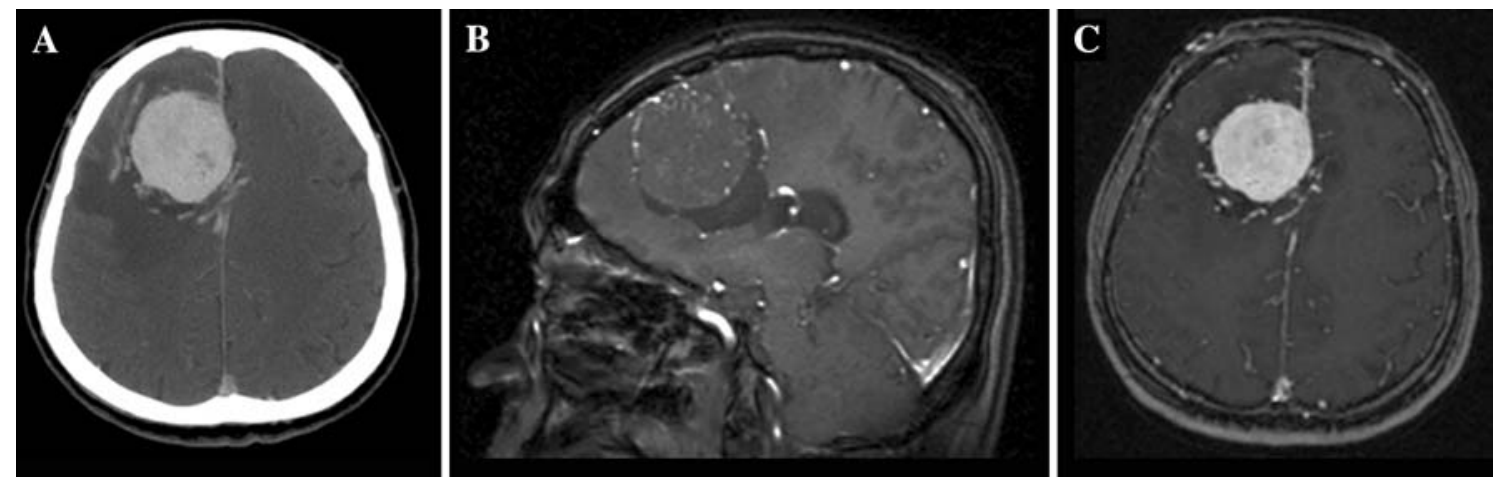

Fig. 1 Pre-operative radiological imaging. a Axial post-contrast CT image of the brain demonstrates a $5 \mathrm{~cm}$ by $5 \mathrm{~cm}$ avidly enhancing mass within the superior right frontal lobe with multiple serpiginous enhancing structures adjacent to the mass consistent with significant vascularity. b \& c Sagittal non-contrast and axial post-contrast MR images again demonstrate a homogeneously enhancing extra-axial mass abutting the right falx with associated extensive tumor related edema and $6 \mathrm{~mm}$ right to left midline shift
Fig. 2 Preoperative angiography. Anteroposterior and lateral preoperative cerebral angiography demonstrating large callosomarginal artery with arterial jet entering the tumor (arrows)
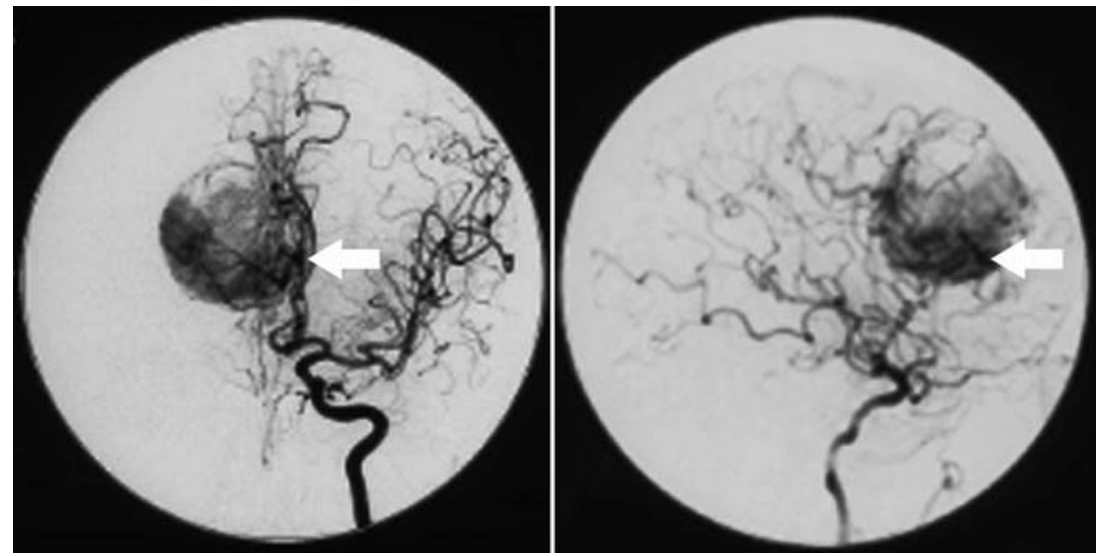
Fig. 3 Three dimensional animation demonstrating surgical approach. a Patient placement with bi-temporal pinning with Mayfield head holder. Lateral view of falcine meningioma with corresponding preoperative angiography shows callosomarginal feeding artery (arrows). b Illustration of cranial vault opening using bipartite bone flap. c Localization and clipping of the right callosomarginal artery feeding branch entering the base of the tumor. d En bloc tumor removal with minimal bleeding or immediate intra-operative complications
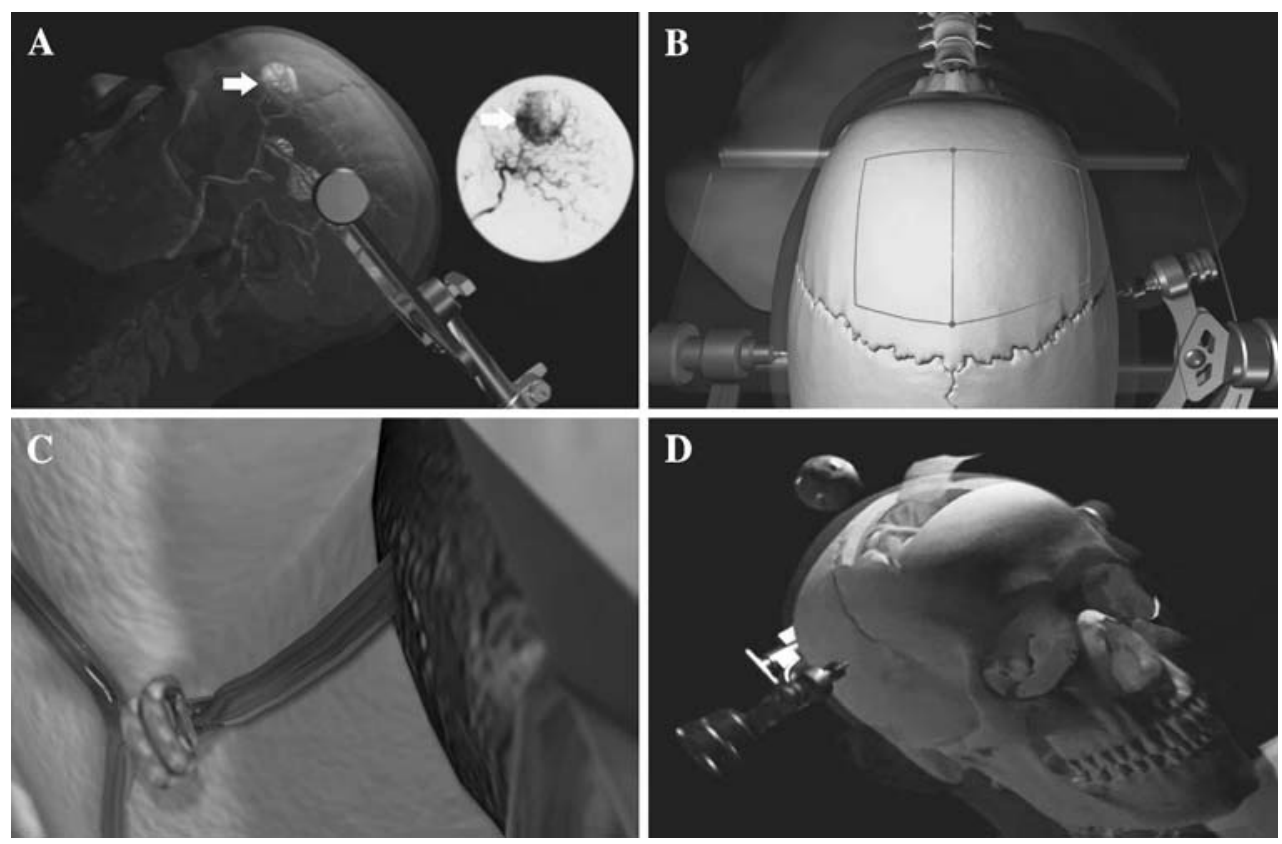

dissected exposing the falx and its free edge. We were able to identify the callosomarginal and pericallosal arteries confirming this with the assistance of an intra-operative navigational system. The inferior aspect of the falx was then incised from its free edge superiorly to follow the callosomarginal artery distally until we could identify the branch directly entering the tumor. As anticipated, the right callosomarginal artery and it's feeding branch entering the base of the tumor was quite large. A $5 \mathrm{~mm}$ permanent straight Yasargil aneurysm clip was placed on the proximal callosomarginal artery branch entering the tumor, and the distal portion was cauterized and divided (Fig. 3c).

Following this, the dura was opened in a U-shaped fashion based medially on the right. Visual inspection revealed no clear way to get around the margins of this tumor without frontal lobe retraction, necessitating a small corticectomy down to the anterolateral and posterior margins of the tumor. Following this a pial dissection plain was developed anteriorly and posteriorly using microscissors, bipolar cautery, and gentle retraction with a Penfield \#1, cauterizing any pial tumor feeders encountered. We encountered brisk bleeding with any attempt to remove the tumor from the falx. For this reason, the superior sagital sinus was ligated anteriorly and posteriorly, taking care to preserve several large parasagital draining veins entering the sinus posteriorly. The corresponding sinus and falx were then excised. Following this, several deep draining veins were identified, cauterized, and divided. The tumor was then removed en bloc with minimal bleeding or immediate intra-operative complications (Fig. 3d). Final pathologic analysis was consistent with WHO grade 1 angioblastic meningioma.
Post-operative outcome

Post-operatively the patient did well and did not show evidence of lower extremity weakness in either the immediate post-operative period, or at 2 year post-operative follow-up. MRI performed on post-operative day two demonstrated gross total resection without evidence of diffusion abnormality to suggest ischemic changes (Fig. 4a-c). He was discharged to home on post-op day 4 without complaint. At 2 year follow-up he was asymptomatic without evidence of recurrent disease on surveillance MRI (Fig. 4d).

\section{Discussion}

Meningiomas are the most common benign intracranial tumor accounting for $13-26 \%$ of all primary intracranial neoplasms with approximately $5 \%$ arising from the falx $[4,5]$. The nidus of falcine meningiomas originally derives its rich vascular network from the dural vasculature and later parasitizes the pial vascular supply as the periphery of the tumor grows [1-3, 5, 6, 10].

Preoperative angiography is often useful in helping plan the surgical approach in cases of falcine meningiomas by delineating the tumor's arterial feeding pattern. Furthermore, cerebral angiography evaluates displacement, of the pericallosal artery and whether arterial branches contribute to the tumor's vascular supply $[1,3,8,9]$. The venous phase can establish whether the tumor has invaded the superior sagittal sinus, determines sinus patency, and provides critical information about the courses of large draining veins [1, 9]. 

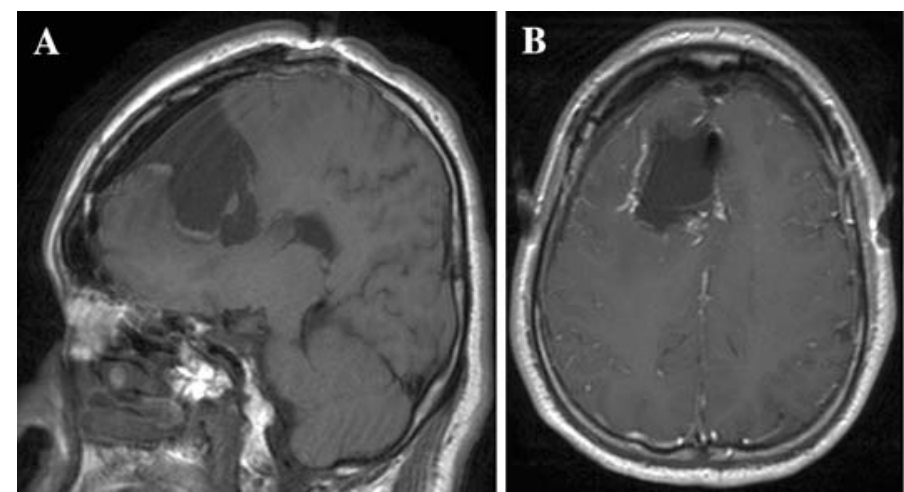

Fig. 4 Post-operative MR images. a-c Sagittal non-contrast, axial post-contrast, and axial diffusion weighted imaging post operative day two show resection cavity with surrounding mild post-operative edema. No post gadolinium enhancement is noted. There is no

In the present case, preoperative embolization was incompletely effective at limiting the tumor's blood supply, largely due to a massive callosomarginal feeding vessel unsuitable for embolization. A direct approach to this artery on the ipsilateral side by dissecting the ill defined arachnoid plane on the anterior surface of the tumor and then retracting the tumor still attached to the falx was thought to be too difficult preoperatively. A contralateral interhemispheric approach was thought to be easier with incision of the lower falx to provide exposure of the artery as it entered the tumor on its inferior surface.

There are other reports of contralateral interhemispheric approaches to intra-axial lesions and intra-ventricular tumors $[4,7]$. The anterior location of this tumor lowered the risk associated with damaging the parasagittal draining veins.

A majority of meningiomas are supplied by ipsilateral meningeal arteries, however, falcine tumors are more likely to also have a contralateral vascular supply. Hattori et al in there study clarifying the characteristics of contralateral blood supply of paramedian meningiomas found that $56 \%$ of falcine meningiomas had a significant degree of contralateral vascular supply [3]. They hypothesize the secretion of angiogenetic factors, such as vascular endothelial growth factor, induce neovascularization, establishment of arterial anastomoses in the falx between the right and left meningeal network, and the subsequent recruitment of contralateral dural arteries [3].

Meningiomas of the anterior two thirds of the falx frequently receive their vascular supply from the anterior falx artery, which itself arises from the ethmoid artery. Since the ethmoid artery arises from the ophthalmic artery there is no way to safely embolize the falcine artery tumor supply.

The prognosis of patients with falcine meningiomas is dependent on the Simpson grade at the time of resection [2]. One case series of 68 patients by Chung et al indicated
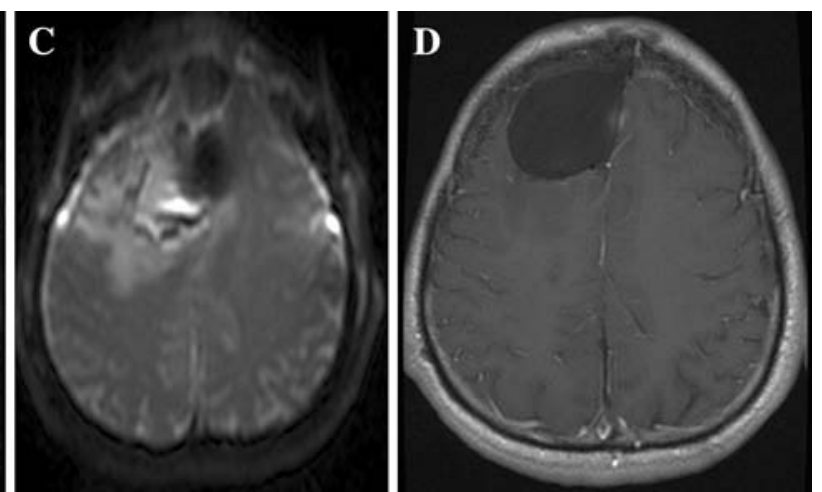

evidence of associated reduced diffusion on diffusion weighted sequences to suggest ischemia. d At 2 year follow up there is no evidence of local recurrence of tumor or other focal areas of abnormal enhancement

the majority of patients who underwent subtotal resection had a tumor that involved the superior sagittal sinus, torcula, or cortical draining vein, were attached firmly to the falx, or were intimately involved with vital arterial structures. This case provides an excellent example of one approach to directly dealing with large, deep interhemispheric feeding vessels unsuitable for embolization allowing for en bloc Simpson grade 1 tumor removal.

In conclusion we present the case of a falcine meningioma with a large callosomarginal feeding branch which was successfully removed following surgical clipping of this vessel via a contralateral interhemispheric approach. Intra-operative control of the vascular supply and complete tumor resection was critical to excellent long term prognosis in this patient. We provide a three dimensional animation of the surgical approach for instructional purposes.

Acknowledgments The first author thanks Bethany Carlson M.S. R.N. for her helpful comments regarding this manuscript. This work was supported by National Institutes of Health National Center for Research Resources Grant TL1 RR024129-01. Computer animation provided by Kenneth Probst, an employee of the Department of Neurological Surgery at the University of California, San Francisco.

Open Access This article is distributed under the terms of the Creative Commons Attribution Noncommercial License which permits any noncommercial use, distribution, and reproduction in any medium, provided the original author(s) and source are credited.

\section{References}

1. Al-Mefty O, Origitano TC, Harkey HL (1996) Controversies in Neurosurgery. Thieme 86-88

2. Chung SB, Kim CY, Park CK, Kim G, Jung HW (2007) Falx meningiomas: surgical results and lessons learned from 68 cases. J Korean Neurosurg Soc 42:276-280. doi:10.3340/jkns.2007.42.4.276

3. Hattori K, Miyachi S, Kobayashi N, Kojima T, Hattori K, Negoro M, Yoshida J, Nagasaka T (2005) Contralateral meningeal artery 
supply of paramedian meningiomas. Surg Neurol 64(3):242-280. doi:10.1016/j.surneu.2005.02.006

4. Lawton MT, Golfinos JG, Spetzler RF (1996) The contralateral transcallosal approach: experience with 32 patients. Neurosurgery 39(4):729-735. doi:10.1097/00006123-199610000-00016

5. Longstreth WT, Dennis LK, McGuire VM (1993) Epidemology of intracranial meningiomas. Cancer 72:639-648. doi:10.1002/ 1097-0142(19930801)72:3<639::AID-CNCR2820720304>3.0.CO; 2-P

6. Louis DN, Scheithauer BW, Budka H, Von Deimling A, Kepes JJ, Kleihues P, Cavenee WK (2000) World health organization classification of tumours. Pathology and genetics of tumours of the nervous system. IARC Press, Lyon, pp 176-184
7. McDermott M (2003) Intraventricular meningiomas. Neurosurg Clin N Am 14(4):559-569. doi:10.1016/S1042-3680(03)00055-X

8. Oyama H, Noda S, Negoro M, Kinomoto T, Miyachi S, Kuwayama M, Kajita Y (1992) Giant meningioma fed by the anterior choroidal artery: successful removal following embolization. Neurol Med Chir (Tokyo) (32):839-841. doi:10.2176/ nmc. 32.839

9. Rossitti S (2007) Preoperative embolization of lower-falx meningiomas with ethylene vinyl alcohol copolymer: technical and anatomical aspects. Acta Radiol 48(3):321-326. doi:10.1080/ 02841850601161547

10. Whittle IR, Smith C, Navoo P, Collie D (2004) Meningiomas. Lancet 363:1535-1543. doi:10.1016/S0140-6736(04)16153-9 